\title{
Investigación para determinar la factibilidad de la constitución de una microempresa productora de mermelada de babaco en el cantón Píllaro
}

\section{Research to determine the feasibility of the establishment of a microenterprise of a babaco jam in the Pillaro canton}

\author{
López Henry ${ }^{1}$ \\ henryrobertlopez@gmail.com \\ Viera Diego $^{2}$ \\ diegosebas07@hotmail.com \\ Bassante Viviana $^{3}$ \\ vibassante@outlook.com \\ Ortiz María $^{4}$ \\ gabyortiz24@hotmail.com
}

Recibido: 1/07/2018, Aceptado: 1/09/2018

\begin{abstract}
RESUMEN
Con las nuevas necesidades de los clientes y el nivel de concientización generado hacia el consumo de productos naturales impulsado por empresarios emprendedores es que el gobierno ha creado varias estrategias para apoyar la inversión de planes de negocios mediante el financiamiento de instituciones financieras públicas. Con ese apoyo y con la necesidad del público meta hacia el consumo de productos nacionales y naturales es que se ha dado paso a un Proyecto de inversión para la implementación de una microempresa procesadora de mermeladas de babaco cuya planta estará ubicada en el cantón Píllaro, por ser el sitio donde existe la mayor producción de babaco. El proyecto contará con 8 departamentos que generará alrededor de 30 puestos de trabajo. Se realizará una inversión inicial de $\$ 379.467,50$, financiando el proyecto con el $84 \%$ de recursos de terceros obtenido del CFN y el $16 \%$ de recursos propios. La empresa realizará la producción de 2 tipos de mermeladas: una de 600 gr y otra de $300 \mathrm{gr}$ con precios de $\$ 1,64$ y $\$ 1,07$ respectivamente. El flujo neto generado para el primer año es de $\$ 572.172,36$ valor que será incrementado al año 5 de $\$ 738.510,39$. La utilidad del ejercicio para el primer año es de $\$ 676.506,96$. El valor actual neto es de $\$ 232.288,47$ con una Tasa Interna de Retorno del $71 \%$ con 0,61 dólares de ganancia por cada dólar invertido en un tiempo de recuperación de un año. Luego de los resultados generados se concluye la aceptación del proyecto siendo factible la inversión.
\end{abstract}

\footnotetext{
1 Docente del Instituto Tecnológico Superior Bolívar. Ecuador

2 Docente del Instituto Tecnológico Superior Bolívar. Ecuador

3 Docente del Instituto Tecnológico Superior Bolívar. Ecuador

4 Docente del Instituto Tecnológico Superior Bolívar. Ecuador
} 
Palabras clave: emprendedores, mercado meta, inversión, utilidad, factible

\begin{abstract}
With the new needs of customers and the level of awareness generated towards the consumption of natural products driven by entrepreneurial entrepreneurs is that the government has created several strategies to support the investment of business plans by financing public financial institutions. With this support and with the need of the target public towards the consumption of national and natural products, an investment project for the implementation of a babaco jam processing microenterprise whose plant will be located in Pillaro, has been given, where there is the largest production of babaco. The project will have 8 departments that will generate around 30 jobs. An initial investment of $\$ 379467.50$ will be made, financing the project with $84 \%$ of third party resources obtained from the CFN and $16 \%$ of own resources. The company will produce 2 types of jams; one of $600 \mathrm{gr}$ and another of $300 \mathrm{gr}$ with prices of $\$ 1.64$ and $\$ 1.07$ respectively. The net flow generated for the first year is $\$ 572,172.36$, which will be increased to year 5 of $\$ 738,510.39$. The profit for the year for the first year is $\$ 676,506.96$. The net present value is $\$ 232,288.47$ with an Internal Rate of Return of $71 \%$ with 0.61 dollars of profit for each dollar invested in a recovery time of one year. After the results generated, the acceptance of the project is concluded and the investment is feasible.
\end{abstract}

Keywords: entrepreneurs, target market, investment, utility, feasible

\title{
Introducción
}

El problema del presente proyecto radica en la escasa variedad de sabores de mermeladas en el mercado generadas por las siguientes causas:

Actualmente, con la era de globalización los clientes están más informados en relación a los productos que se comercializan, por lo cual sus gustos y preferencias cambian frecuentemente. Por otra parte, los cambios climáticos y la ubicación estratégica de nuestro país hacen que el cultivo para cada tipo de frutas sea diferente porque cada una de ellas depende de la estación del año en la que se encuentre. Lo que daría lugar al volumen inconstante de producción, perdiendo ventas y utilidades de la empresa. Y como última causa, el libre acceso de productos importados que se han posicionado tanto en la mente de los consumidores que es casi imposible lograr que opten por adquirir un producto ecuatoriano.

Indicadores sociales

En Tungurahua, la provincia ocupa el primer lugar en la producción de babaco con el $60 \%$ de la producción nacional, con 1841 toneladas por año. El cantón Patate es considerado la meca de esta fruta. De acuerdo a información proporcionada por un agricultor de la zona, cada semana saca alrededor de 20 cajones de babaco de 1.500 plantas en alrededor de $3.000 \mathrm{~m}^{2}$ de terreno (El Productor, 2011).

Por otra parte, los cambios climáticos y la ubicación estratégica de nuestro país que puede jugar un papel importante y por otra un aporte negativo hace que el cultivo 


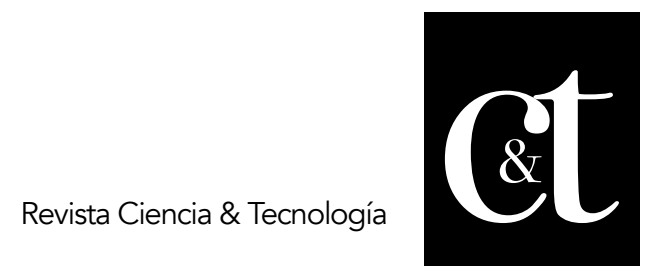

No. 20, 31 de octubre de 2018

ISSN impreso: 1390 - 6321

para cada tipo de frutas sea diferente porque cada una de ellas depende de la estación del año en la que se encuentre. Lo que daría lugar al volumen inconstante de producción, perdiendo ventas y utilidades de la empresa.

Ubicación Geográfica

Píllaro es un cantón ubicado en la provincia de Tungurahua que posee un enorme potencial agrícola debido a que cuenta con suelos fértiles, fuentes de agua puras, variados climas por efecto de los pisos altitudinales y nichos ecológicos, teniendo así lugares aptos para el sembrío y la cosecha de tubérculos; frutas como la pera, manzana, tomate de árbol, granadilla, babaco y la claudia forman parte importante de la producción agrícola (GADM Píllaro, 2014).

Datos generales del babaco

El babaco, conocido como la papaya de la montaña, originaria de los valles subtropicales del Ecuador, es un arbusto pequeño, con hojas de variadas formas triangulares y sus flores tienen forma acampanada con pétalos blancos y amarillos. El fruto tiene forma alargada de $20 \mathrm{~cm}$ de largo por $5 \mathrm{~cm}$ de ancho. Cuando la fruta está madura, la piel es amarilla, intensa y la pulpa es de color amarillento. Esta fruta tiene un aroma exquisito, con un sabor delicado y fresco; es jugosa y un poco ácida. Es generalmente cultivada en Ecuador desde antes de la conquista española y se ha convertido en una fruta de consumo tradicional en la zona centro del país; donde es preparada en jugo, variedad de dulce y consumo fresco. Por ser una fruta sin semilla y con cáscara delgada, presenta buenas características para su consumo e industrialización (Mesías, 2012).

A nivel mundial se ha aumentado la demanda de la fruta exótica, donde el babaco es uno de ellos por sus características en el sabor y por el nivel proteico. La variabilidad de climas con las que cuenta el país, le ha permitido ser reconocido por su riqueza biológica, ubicándole entre los países con la más amplia biodiversidad por unidad de superficie a nivel global. Como dato resaltable, el mercado alemán se ha constituido como uno de los más importantes por el poder adquisitivo de Europa. En el país existen alrededor de 252 hectáreas sembradas por esta fruta bajo invernadero o a cielo abierto (Espinosa, 2016).

En Ecuador, el babaco fue introducido en 1985, pertenece a la familia de las caricáceas, es originaria de las zonas andinas del Ecuador y Colombia. Es cultivada en zonas donde no existen vientos ni heladas fuertes. Generalmente son cultivados en los valles de la región interandina de las provincias: Imbabura, Pichincha, Tungurahua, Chimborazo, Azuay y Loja.

En el cantón Píllaro cuenta con gran cantidad de producción de babaco, aspecto positivo para el desarrollo del presente proyecto, por lo que resulta factible implementar una microempresa procesadora de mermelada, para mejorar el movimiento económico del cantón y la provincia, generar fuentes de empleo e innovar la producción tungurahuense. 


\section{Comercio exterior}

El cultivo de babaco presenta una de las tasas de retorno más importantes dentro de los frutales, porque pueden alcanzar un promedio de 225 toneladas métricas por hectárea, durante el período de 2-3,5 años. Las expectativas de exportación que posee el agricultor han aumentado la necesidad de propagación, sin embargo, su forma de reproducción, la multiplicación del babaco se hace mediante estacas, porque el fruto no produce semilla. Debido a su producción escalonada durante el año, posee una ventaja comparativa con una alta tasa de retorno (Rodríguez, 2013).

De acuerdo al Banco Central del Ecuador en el 2012, se exportaron 2.351,43 toneladas de babaco, con un precio de exportación de 1,77 dólares americanos.

Tabla $N^{\circ} 1$. Exportaciones de babaco ecuatoriano

\begin{tabular}{|c|c|c|c|}
\hline \multicolumn{4}{|c|}{ Exportaciones } \\
\hline Año & Toneladas & FOB & $\begin{array}{c}\text { Precio } \\
\text { Referencial }\end{array}$ \\
\hline 2012 & 2351,43 & $\$ 4161.12$ & $\$ 1.77$ \\
\hline
\end{tabular}

Fuente: Banco Central del Ecuador (2015)

\section{Método}

La presente investigación se efectuó de forma profunda en el cantón Píllaro en la cual se analizaron los productores y expendedores de fruta (babaco). $Y$ en relación al cantón Ambato se investigó a los consumidores, es decir al mercado meta. El resultado de la encuesta permitió tener una clara idea de la situación actual de la oferta y demanda de mermeladas en el mercado, su distribución, precios y presentaciones. El mercado al que se pretende atender, son locales mayoristas, para reducir el costo y expenderlos al consumidor final obteniendo una ligera ganancia por la transacción del producto. Mencionado aquello, el mercado al que se pretende llegar es:

- Supermercados

- Micro mercados

- Tiendas 
Tabla $\mathbf{N}^{\circ} 2$. Dimensión geográfica consumidor final

\begin{tabular}{|l|l|}
\hline \multicolumn{1}{|c|}{ Variable } & \multicolumn{1}{c|}{ Descripción } \\
\hline País & Ecuador \\
\hline Región & Sierra \\
\hline Provincia & Tungurahua (504.583) \\
\hline Ciudad & Ambato (329.856) \\
\hline
\end{tabular}

Fuente: Investigación de campo

\section{Resultados}

Estudio de mercado

Se analizaron los productores y expendedores de fruta (babaco) en el cantón Ambato, se decidió atender a tiendas, minimarkets y supermercados con el objetivo de minimizar costos por la expedición del producto final al consumidor.

Bajo estas características, el mercado objetivo el cual se tiene en la mira representa los 2.842 centros (tiendas, minimarkets y supermercados), estos datos fueron procesados de la base de datos del Servicio de Rentas Internas (2017) teniendo como resultado final: tiendas (2.729), minimarkets (107) y supermercados (6) en la ciudad de Ambato.

\section{Tabla N³. Mercado Objetivo}

\begin{tabular}{|l|r|r|}
\hline \multicolumn{1}{c|}{ Variable } & Descripción & Porcentaje \\
\hline Tiendas & 2729 & $96,02 \%$ \\
\hline Minimarket & 107 & $3,76 \%$ \\
\hline Supermercados & 6 & $0,21 \%$ \\
\hline Total & $\mathbf{2 8 4 2}$ & $\mathbf{1 0 0 , 0 0 \%}$ \\
\hline
\end{tabular}

Fuente: Base de datos del SRI (2017)

Mercado objetivo y segmentación

El mercado objetivo está constituido por 2.842 centros entre tiendas, minimarkets y supermercados y fue necesario obtener una muestra para la aplicación del instrumento diseñado y se determinó que 338 intermediarios.

Productos a ofertar

El babaco, al igual que la papaya, no es un árbol sino una planta imperecedera grande que se asemeja a una palma pequeña. Es un frutal nativo de los valles subtropicales de la región interandina ecuatoriana, que se dan en las zonas de Baños, Ambato y Tumbaco. Su fruto tiene varias características muy apetecibles, es muy bajo en calorías, la pulpa que posee es cremosa sin semillas y corteza muy fina que puede ser comestible. 
Tabla $N^{\circ} 4$. Oferta en productos (frecuencia de compra)

\begin{tabular}{|c|c|c|c|c|}
\hline Descripción & Frecuencia & Porcentaje & $\begin{array}{c}\text { Unidades } \\
\text { ofertadas de } \\
\text { producto }\end{array}$ & $\begin{array}{c}\text { Unidades } \\
\text { ofertadas de } \\
\text { producto anual }\end{array}$ \\
\hline Mensual & 2 & $1 \%$ & 3.536 & 42.431 \\
\hline Trimestral & 10 & $3 \%$ & 17.680 & 70.719 \\
\hline Semestral & 326 & $96 \%$ & 576.361 & 1.152 .722 \\
\hline Total & 338 & $100 \%$ & 597.577 & 1.265 .873 \\
\hline
\end{tabular}

Fuente: Encuesta aplicada

El valor nutricional del babaco por $100 \mathrm{~g}$ de fruta se detalla en la siguiente tabla:

\section{Tabla $N^{\circ} 5$. Valor nutricional}

\begin{tabular}{|l|l|}
\hline Agua & $95 \mathrm{~g}$ \\
\hline Vitamina C & $28 \mathrm{mg}$ \\
\hline Vitamina E & $27 \mathrm{mg}$ \\
\hline Niacina & $0.5 \mathrm{mg}$ \\
\hline Riboflavina & $0.02 \mathrm{mg}$ \\
\hline Tiamina & $0.03 \mathrm{mg}$ \\
\hline Proteína & $0.7 \mathrm{~g}$ \\
\hline Potasio & $165 \mathrm{mg}$ \\
\hline Calcio & $13 \mathrm{mg}$ \\
\hline Fósforo & $7 \mathrm{mg}$ \\
\hline Grasa & $0.2 \mathrm{~g}$ \\
\hline Azufre & $12 \mathrm{mg}$ \\
\hline Piridoxina & $0.05 \mathrm{mg}$ \\
\hline Lípidos & $0.1 \mathrm{~g}$ \\
\hline Fibra Alimentaria & 1.1 \\
\hline Sodio & $1 \mathrm{mg}$ \\
\hline Carbohidratos & $6 \mathrm{~g}$ \\
\hline Calorías & $8 \mathrm{mg}$ \\
\hline Ácido Ascórbico & 31 \\
\hline Carotenos & $0.09 \mathrm{mg}$ \\
\hline Hierro mineral & $0.3 \mathrm{mg}$ \\
\hline
\end{tabular}

Fuente: Proyecto de Elaboración de mermelada de babaco como producto no tradicional de exportación al mercado europeo (2004)

Precio

Según el levantamiento de información se considera que el precio promedio de venta en el mercado de la mermelada debería ser de $\$ 2,20$. 


\section{Beneficiaros}

Los beneficiarios directos la constituyen la ciudadanía en general, quienes requieren de un producto para darle mayor valor en su consumo. Por otra parte, los beneficiarios indirectos serían los profesionales o las persona que requieren de empleo, porque se apertura rían fuentes de trabajo.

Determinación de precio para el proyecto

Estimación del presupuesto

Tabla N6. Presupuesto

\begin{tabular}{|l|l|}
\hline Descripción & Valor \\
\hline Maquinaria y Equipo & $\$ 10.318,85$ \\
\hline Materia prima & $\$ 633211,94$ \\
\hline Mano de obra & $\$ 11.130,00$ \\
\hline TOTAL & $\$ 654660,79$
\end{tabular}

Fuente: Investigación de campo

Estudio de la demanda

Para la estimación de la demanda del producto y del proyecto y en la constitución de una planta para su fabricación, se aplicó una encuesta para determinar la aceptación del producto.

Para efectuar el cálculo respectivo de la demanda en personas (intermediarios) y determinar la aceptación del producto (mermelada de babaco) es necesario tomar como referencia el porcentaje que respondió de forma positiva a la pregunta donde se menciona si estaría dispuesto a comprar mermelada de babaco para ser comercializado y se trabajó con base a la Tasa de Crecimiento Poblacional (TCP) $1,28 \%$ al 2018.

Una vez desarrollado los cálculos adecuados se establece que la demanda en personas (intermediarios) para el año 2018 será de 1.976 centros intermediarios y cada año experimentará una variación de $1,28 \%$ proyectando una demanda en personas (intermediarios) para el año 2022 de 2.079 centros intermediarios quienes estarán dispuestas a comprar el producto (mermelada de babaco) producido por la empresa.

Demanda de productos

Se proyecta que la demanda en productos para el año 2018 será de 2.888.156 fundamentados en la cantidad y frecuencia de compra, una vez establecido este valor, para la proyección de los años posteriores se trabajó con la tasa de crecimiento poblacional vigente que es del 1,28\%, es decir para el año 2022 la demanda en 
productos será de 3.038 .893 productos (unidades).

Estudio técnico

El principal factor para la localización de la planta es la disponibilidad de materia prima. Por lo cual una de las principales ciudades es Píllaro donde se produce babaco en proporciones grandes, es fundamental indicar que todas las parroquias poseen un espacio físico apto para la instalación de la planta procesadora de mermelada, sin embargo, la parroquia que resalta un óptimo espacio físico es San José de Paoló y en todos los factores.

Tabla N7. Factores de Localización

\begin{tabular}{|l|l|l|}
\hline Nro & Factor & Peso \\
\hline 1 & $\begin{array}{l}\text { Cercanía de los principales } \\
\text { centros de consumo }\end{array}$ & 0,30 \\
\hline 2 & Disponibilidad de materia prima & 0,10 \\
\hline 3 & Espacio físico & 0,30 \\
\hline 4 & Clima & 0,15 \\
\hline 5 & Estímulos fiscales & 0,15 \\
\hline \multicolumn{2}{|c|}{ Total } & $\mathbf{1 , 0 0}$ \\
\hline
\end{tabular}

Fuente: Investigación de Campo

Proceso productivo

El gráfico 1 muestra el equipamiento de la planta donde también se establece el proceso de producción a utilizar. 


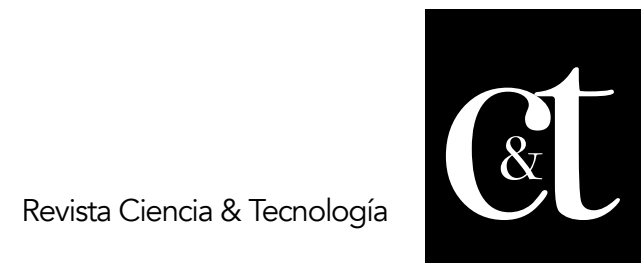

No. 20, 31 de octubre de 2018

ISSN impreso: 1390 - 6321

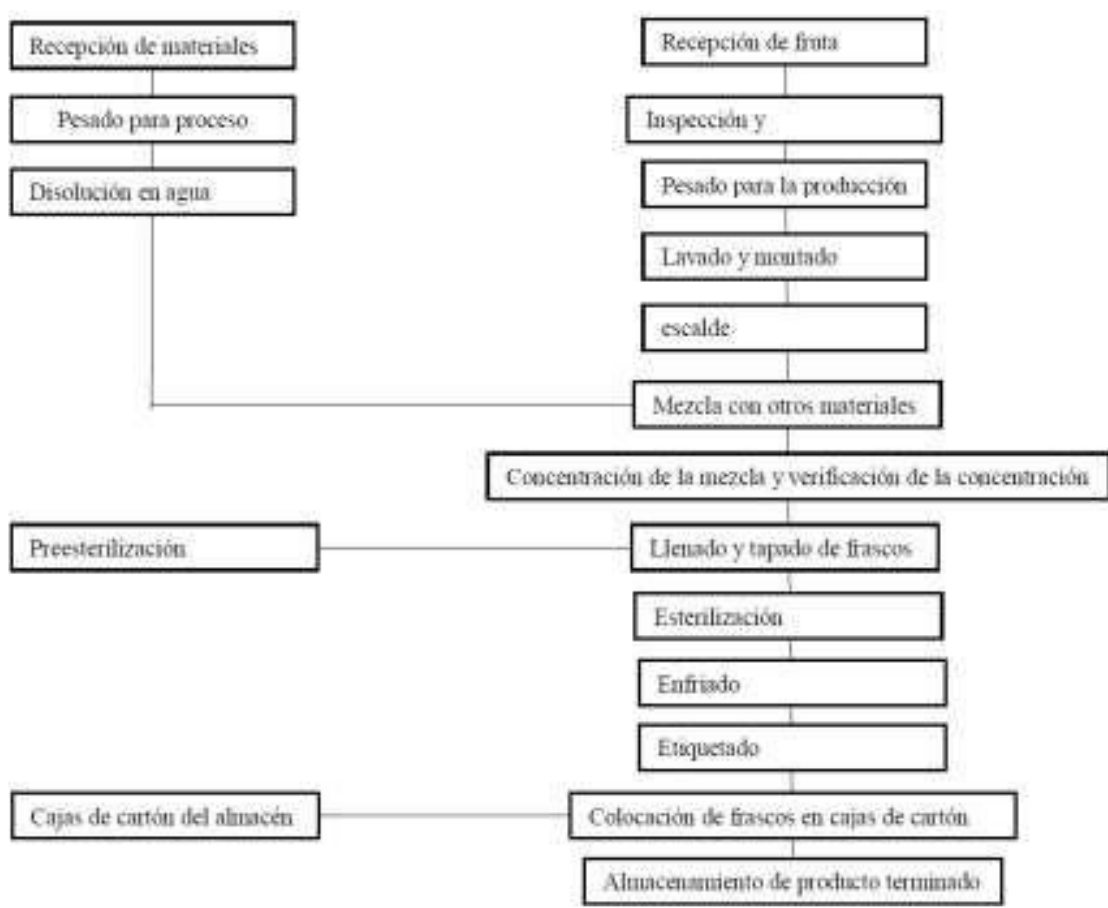

\section{Gráfico 1. Equipamiento de la planta}

Fuente: Elaboración propia

Costo en maquinaria, materiales y herramientas

El costo en maquinaria tiene un total de $\$ 10.318,85$, distribuido para la compra de básculas, montacargas, lavadora de agua a presión con bomba de $5 \mathrm{HP}$, preesterilizadora de vapor tipo túnel y en un purificador de agua de carbón activado. 
Tabla $N^{\circ} 8$. Costos

\begin{tabular}{|l|l|}
\hline \multicolumn{2}{|l|}{ Materia Prima Directa para Frascos de 600 Gramos } \\
\hline Costo Unitario Anual & $\$ 0,7228176$ \\
\hline Materia Prima para Frascos de 300 Gramos \\
\hline Costo Unitario & $\$ 328.332,12$ \\
\hline Costo Anual & $\$ 0,4818784$ \\
\hline Mano de obra & $\$ 304.879,82$ \\
\hline Mano de obra administrativa & $\$ 1.900,00$ \\
\hline Mano de obra operaria & $\$ 9.230,00$ \\
\hline $\begin{array}{l}\text { Con un total de 29 personas el global } \\
\text { de salarios es de }\end{array}$ & $\$ 11.130,00$ \\
\hline
\end{tabular}

Fuente: López et al. (2018)

Estudio administrativo y jurídico

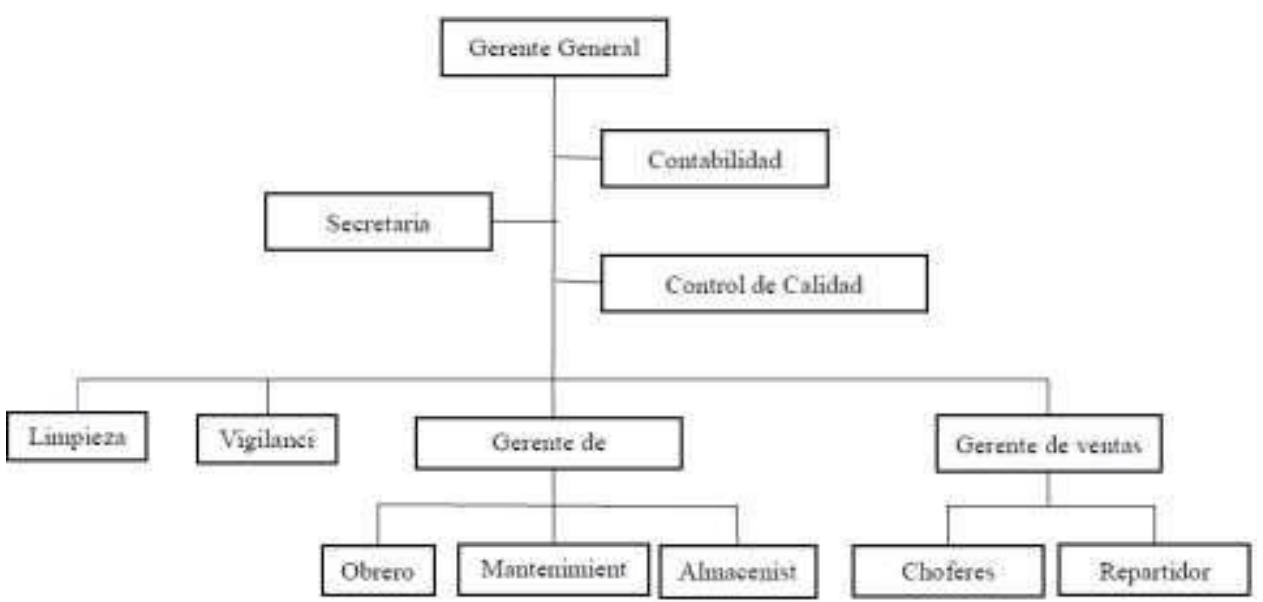

Gráfico 2. Estudio organizacional

Fuente: López et al. (2018) 
Estudio Financiero

Tabla N9. Balance General

\begin{tabular}{|l|l|}
\hline Activo & 38061,75 \\
\hline Total, activo corriente & 376210,00 \\
\hline Total, fijo tangible & 4407,50 \\
\hline Fijo intangible & 418679,25 \\
\hline Total, activo & 350000 \\
\hline Pasivo & 68679,25 \\
\hline Total, préstamo & 418679,25 \\
\hline Total, patrimonio &
\end{tabular}

Fuente: López et al. (2018)

Inversión inicial

La inversión inicial para el presente estudio ostenta un valor total de \$379.467,50 y se encuentra constituido por un local que es un terreno por un valor de $\$ 18.000$, instalaciones y remodelaciones con un valor de $\$ 170.515,05$; muebles y enseres con un valor de $\$ 10.280,00$; maquinaria y equipo con un valor de $\$ 124.888,85$; vehículos con un valor de $\$ 33.000,00$, equipo de computación con un valor de $\$ 19.526,10$, inventario con un valor de $\$ 2.681,00$ y finalmente, los costos de constitución que tienen un valor de $\$ 1.726,50$.

Forma de financiamiento

El valor total de la inversión inicial para el proyecto de una empresa productora de mermelada de babaco es de $\$ 418.679,25$, con un $84 \%$ financiado con la CFN, que asciende a un valor de $\$ 350.000,00$ con una tasa de interés anual de $11,71 \%$ y un $14 \%$ de recursos propios que será dividido entre todos los involucrados de este proyecto, dando prioridad a la participación de acciones a los productores de babaco, este valor será de \$ \$68.679,25.

Evaluación Financiera

Tabla $N^{\circ} 10$. Estructura financiera

\begin{tabular}{|c|c|c|c|c|}
\hline TMAR & VALOR & $\begin{array}{l}\text { ESTRUCT } \\
\text { URA }\end{array}$ & $\begin{array}{c}\text { COSTO DE } \\
\text { OPORTUNI } \\
\text { DAD }\end{array}$ & $\begin{array}{c}\text { PONDERA } \\
\text { CIÓN }\end{array}$ \\
\hline $\begin{array}{l}\text { RECURSOS } \\
\text { PROPIOS }\end{array}$ & $\begin{array}{l}\$ \\
68.679,25\end{array}$ & $16 \%$ & $5,32 \%$ & $0,87 \%$ \\
\hline $\begin{array}{l}\text { RECURSOS } \\
\text { AJENOS }\end{array}$ & $\begin{array}{l}\$ \\
350.000,00\end{array}$ & $84 \%$ & $11,71 \%$ & $9,79 \%$ \\
\hline TOTAL & $\$$ & $100 \%$ & $17,03 \%$ & $10,66 \%$ \\
\hline
\end{tabular}

Fuente: López et al. (2018)

El costo promedio ponderado CCPP que engloba una sola cifra expresada en 
porcentaje donde a efectos de la estructura del endeudamiento en este caso un 90/10 influirá en el monto final con las tasas de interés y efectos fiscales a noviembre del 2017 por lo que vale la pena tomar el tiempo necesario en hacer un estudio de banca para analizar los porcentajes de la tasa activa y pasiva, en fin en el presente estudio el Tmar es de 10,66\%; dato que permite interpretar que la TIR debe ser mayor a esta mínima base para que el retorno sea beneficioso.

Tal cual como se explicó anteriormente en el flujo de caja, es importante analizar el resultado final del flujo anual, para en función de ello saber qué año es el de superávit o deficiencia, por ende, se procede a calcular la TIR y los evaluadores más importantes:

Tabla No.11. Evaluación financiera

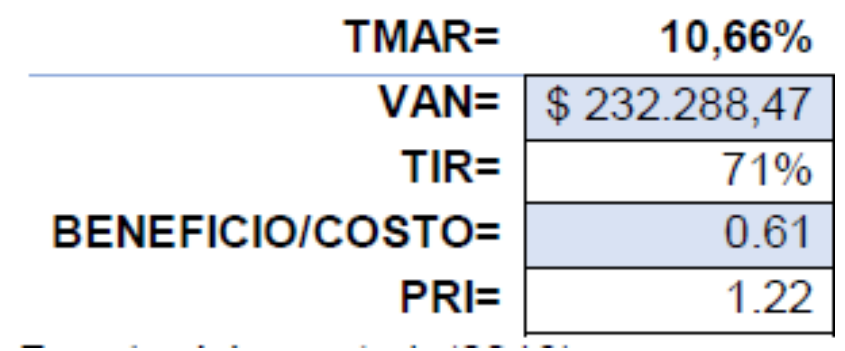

Fuente: López et al. (2018)

Se obtiene una tasa interna de retorno del $71 \%$ que al ser mayor al costo promedio ponderado o TMAR (tasa mínima de retorno) se indica que el proyecto es factible y rentable. Por otro lado, el valor actual neto es de \$232.288,47; lo que quiere decir que al ser mayor de la inversión inicial y al ser mayor a cero el proyecto se acepta.

La relación costo beneficio, es la relación entre la prima o la ganancia extraordinaria, y la sumatoria del valor presente de los egresos, se concluye que la ganancia extraordinaria o prima por cada unidad monetaria invertida es de $\$ 0,61$ unidades actuales monetarias.

\section{Conclusiones}

El proyecto se determinó factible debido a la zona donde se ubicará la planta y por la inversión y el financiamiento para la obtención de recursos para la constitución de la organización.

La planta será constituida como una organización que cuenta inicialmente con 29 personas que ocuparan cargos operarios y administrativos con una estructura empresarial definida de manera funcional y legal de la planta productora.

Se realizó un estudio de mercado donde se determinó el precio del producto y la cantidad de producción con una proyección mensual y anual. 


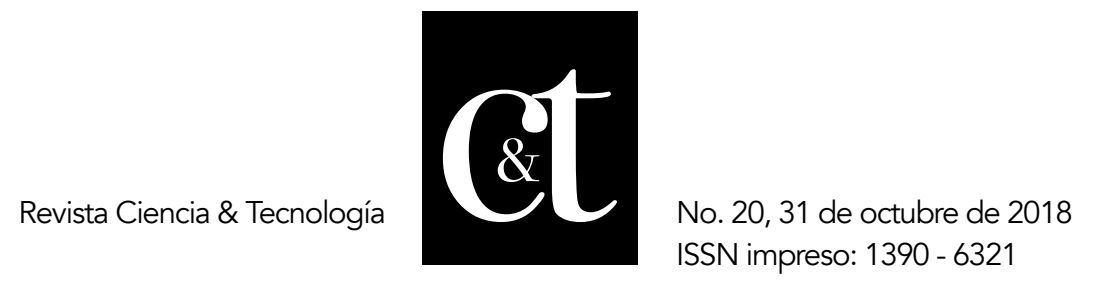

\section{Referencias bibliográficas}

Banco Central del Ecuador (2015). Estadísticas económicas. Quito, Pichincha, Ecuador.

El Productor. (24 de Octubre de 2011). El babaco es un cultivo exótico bien apetecido. Guayaquil, Guayas, Ecuador.

Espinosa, K. (2016). Evaluación agronómica del babaco (Carica pentagona) con dos fertilizantes químicos en diferentes dosis en el cantón Pangua. La Maná, Cotopaxi, Ecuador. Revista UTCiencia, Vol. 3 No.2: 129-135.

GADM Píllaro (2014). Plan de Desarrollo y Ordenamiento Territorial Del Cantón Santiago de Píllaro. Píllaro: Geoingenieria.

Mesías, J. (2012). Proyecto de factibilidad para la producción de babaco bajo invernadero y su comercialización en la ciudad de Quito. Quito, Pichincha, Ecuador.

Rodríguez, K. (2013). Plan de exportación de babaco para la compañía agroproductos del Ecuador "El migrante CIA". Tesis, Universidad Técnica Particular de Loja.

Servicio de Rentas Internas (2017). Base de datos de empresas. https://www.sri.gob.ec/web/guest/catastros 